\title{
Scalable Intersection based Vehicular Ad-hoc Network Supporting QOS
}

\author{
Priya Chanana \\ Department of Computer Science and Engineering \\ P.I.E.T, Kurukshetra University, Haryana 132102. \\ India.
}

\author{
Nishant Prabhat \\ Department of Computer Science and Engineering \\ P.I.E.T, Kurukshetra University, Haryana 132102. \\ India.
}

\begin{abstract}
Real-time safety and non-safety applications for vehicular ad hoc networks (VANETs) requires understanding of the network topology characteristics since these parameters determine both the performance of routing protocols and the feasibility of an application over VANET. VANET support a large spectrum of mobile distributed applications that range from traffic alert dissemination and dynamic route planning for context-aware advertisement and file sharing.

In this paper, we investigate the use of vehicle-to-vehicle (V2V) communications to enable the navigation of traffic intersections and to mitigate collision risks so that to increase intersection throughput significantly. We will focus on City Roads Using Real-Time Vehicular Traffic and compared them with protocols representative of mobile ad hoc networks and VANETs. Simulation will be done using urban city maps settings and they will evaluate performance best in terms of average delivery rate.
\end{abstract}

\section{Keywords}

Vehicular ad hoc networks, RSU Communications, Mobility.

\section{INTRODUCTION}

Wireless Technology are used by millions of people. They use this for Internet admission alongside our laptop, for data transfer amid phones, and even for entertainment. An ad-hoc web can rise both the scope and the whole coverage span of the network [1]. There are disparate technologies and protocols obtainable that are suitable for the use in ad-hoc networks. There are different technologies and protocols available that are suitable for the use in ad-hoc networks. Particularly well suited is the IEEE 802.11 standard that meets all requirements for a use in ad hoc mode. Today's Wi-Fi standards have a sufficient transmission range and a high data rate. Upcoming Wi-Fi standards will achieve data rates, which have never been possible previously. But even older standards like Bluetooth have ad hoc capabilities that can be useful for wireless data exchange.

But the transmission of data includes hurdles like noise, fading and interference and this result into the reduction of bandwidth as compared to wired connection and overhead increase when data is transmitted along multiple hops in an ad-hoc network. Before the technologies are presented in detail, they should define the term "ad hoc network" [2]. The differences between mobile ad hoc networks and mesh networks should be made clear as well. Wireless communication between mobile nodes.

- Nowadays there are varied data-rate-intensive demands that are reliant on broadband and reliable networks. Concrete examples for this kind of demands that ought to benefit from ad hoc networking are: multimedia conferencing, video streaming, web storage, file transfer, transmission of HDTV signals, transmissions of countless audio signals and online gaming[3]. Our main aim is to profit from Ad-Hoc Networks. Ad Hoc webs can additionally be utilized to furnish internet connection to spans lacking groundwork employing wireless

\section{RELATED WORK}

International Symposium on High Assurance Systems Engineering2015 IEEE[17]Traffic light is considered as one of the most competent methods to alleviate traffic congestion and carbon emission problems. Though, established traffic light cannot encounter the trials in traffic regulation acted by the fast producing number of vehicles and rising intricacy of road conditions. So we use a knowledge shouted vibrant traffic regulation method established on adjacent traffic light (VTL) for Vehicular Ad-hoc Network.

International Journal of Digital Information and Wireless Communications (IJDIWC) 3,(2013)[5] Vehicular Ad-hoc web is an growing class of wireless web that furnish effectual contact amid vehicles and amid vehicles and road side units. VANETs are obtaining attention from both manufacturing and intellectual point of think due to its requests fluctuating from protection requests to infotainment applications. In this paper, they ponder how traffic lights alter the design of routing protocol in city vehicular communication. In city nature the roads are partitioned to countless segments due to the traffic lights. The design of a routing protocol in such an nature is difficult. This paper proposes a new intersection established routing protocol that seizes traffic lights in to thought and alongside low overhead.

In Vehicular Technology Conference (VTC Spring), 2013 IEEE 77th, pp. 1-5. IEEE, 2013. [6]VANET can discriminated from supplementary kinds of ad-hoc webs, such as Mobile Ad-hoc Webs (MANETs), by factors like elevated mobility and decreased communications. This paper proposes TIGER, a new geographical routing protocol for city environments. This protocol uses real period innate traffic data at intersections to enhance the presentation of routing by selecting reliable road-based routes. The protocol is strengthened by a neighborhood-based intersection detection mechanism that ease the protocol from the demand for a road map.

International Journal in Wireless Systems 2, no.1 (2013). [7] Vehicular Ad-hoc web can be believed as an growing class of wireless web that furnish effectual contact amid vehicles and amid vehicles and road side units. Design of an effectual routing protocol is tough due to recurrent topology adjustments and recurrent web partition. Survey of assorted 
routing protocols is vital for intelligent Intelligent Transport Arrangement (ITS).

Intelligent Transportation Systems, IEEE Transactions on 14, no. 1 (2013): 199-213. [18]Vehicular ad hoc networks (VANETs) have become viable and valuable for their wide variety of novel applications, such as road safety, multimedia content sharing, commerce on wheels, etc. Apart from this, by tracking the movement of source as well as destination, the back-bone nodes enable a packet to be forwarded in the changed direction. Simulation results signify the benefits of the proposed routing strategy in terms of high packet delivery ratio and shorter end-to-end delay. We propose a hop greedy routing protocol that aims to reduce the end-to-end delay by yielding a routing path that includes the minimum number of intermediate intersections. The zone wise partitioning of a city road network is an important design framework for the efficient functioning of the destination discovery procedure.

\section{PROPOSED WORK}

VEHICULAR ad hoc webs (VANETs) are anticipated to prop a colossal spectrum of mobile distributed requests that scope from traffic alert dissemination and vibrant path arranging to context-aware advertisement and file sharing. Thinking the colossal number of nodes that give in these webs and their elevated mobility, the setback yet continue concerning the feasibility of requests that use end-to-end multi-hop contact in Intersection Routing on Metropolis Roads after they are gave in Real-Time Vehicular Traffic. The main concern is whether the presentation of VANET routing protocols can gratify the throughput and stay necessities of such applications. Our work focuses on VANET routing in city-based scenarios.

We will design and apply an enhanced ideal for Intersection Instituted VANET Routing on Metropolis Roads Employing Real-Time Vehicular Traffic and contrasted them alongside protocols representative of mobile ad hoc webs and VANETs. Simulation will be completed employing city metropolis charts settings and they will assess presentation best in words of average transport rate.

\section{METHDOLOGY}

\section{Routing in Ad hoc networks}

There are hundreds of routing protocols that have been counseled for ad hoc networks. In MANET no fixed web topology is used. Therefore, mobile nodes accept each runtime topology due to their own vibrant behavior. In supplement, there exists not even solitary method for routing in MANET, as web is crafted at runtime. . So routing protocols for MANET is one of the challenging spans due to its vibrant and ad hoc nature [5]. Due to these setbacks countless routing protocols have been industrialized that can encounter the vibrant nature of ad hoc network. These disparate routing protocols are shouted as topology established routing. Further, in this chapter they focus on topology established routing protocols. These protocols and their kinds were extensively learned in order to judge concerning their suitability in VANET.

\subsection{Factors Affecting Mobility In Vanets}

The mobility outline of nodes in a VANET influences the path invention, maintenance, reconstruction, consistency and caching mechanisms. At each instant, a VANET can have both static (non-moving) and vibrant (moving) nodes. The static nodes incline to dampen the adjustments in topology and routing by replacing as stable relaying points for packets to/from the bordering nodes. On the supplementary hand, vibrant nodes add entropy to the arrangement and cause recurrent path setups, teardowns, and packet losses. This chapter debates the result of assorted factors that impact the mobility outline in VANETS.

\section{Layout of streets}

Streets power nodes to confine their movements to well described paths. This constrained movement outline determines the nodes' spatial allocation and the network's connectivity. This restricted movement highlights the meaning of factors such as the nodes' transmission scope, because the layout of the roads could be such that vehicles travelling on parallel roads spaced distant separately could be out of contact range. Roads can have whichever solitary or several lanes and can permit whichever one-way or two-way traffic

\section{Block size}

Urban spans are normally tear into blocks of assorted sizes. A metropolis block can be believed the smallest span encircled by streets. These blocks can be of disparate sizes metropolitan spans usually have tinier metropolis blocks than tinier towns. The block size dictates the density of the intersections in that span, that in coil determines the frequency alongside that a vehicle stops. It additionally determines whether nodes at bordering intersections can hear every single other's wireless transmissions. Larger blocks should rise the network's sensitivity to vehicles clustering at intersections and to web partitioning, and consequence in a degraded presentation.

\section{Traffic control mechanisms}

The most public traffic manipulation mechanisms at intersections are halt signals and traffic lights. A vehicle needs to halt at a red light till it turns green. A vehicle additionally needs to halt at a halt signal for a insufficient seconds beforehand advancing onward. These mechanisms cause the formation of clusters and queues of vehicles at intersections, subsequently cutting their average speed. Decreased mobility implies extra static nodes and slower rates of path adjustments in the network. On the supplementary hand, cluster formation can additionally adversely alter web presentation alongside increased wireless channel contention and increased web partitioning.

\section{Interdependent vehicular motion}

Vehicles cannot ignore physical constraints acted by the attendance of roads and adjacent vehicles. Every single vehicle's movement is affected by the movement outline of its encircling vehicles. For example, a vehicle needs to uphold a minimum harmless distance from the one in front of it, rise or cut its speed, or change to one more lane to circumvent congestion.

\section{Average speed}

The speed of the vehicle determines how swiftly its locale adjustments, that in coil determines the rate of web topology change. The speed check of every single road determines the average speed of vehicles and how frequently the continuing paths are broken or new paths are established. Additionally, vehicles' acceleration/deceleration and the map's topology additionally alter their average speed - if a chart has less intersections, it implies that its roads are longer, permitting vehicles to move at higher speeds for longer eras of time.

\section{Simulation of VANET Applications}

Vehicle-to-vehicle contact is an extremely actual and challenging topic. Vehicles outfitted alongside mechanisms capable of short-range wireless connectivity can form a 
particular mobile ad-hoc web, VANET-Vehicular Ad-hoc Network. Finished features of VANET simulators are presented. They delineate a design that integrates the early two models and permits a facile interfacing alongside the third model. Features concerning the web / contact ideal are additionally given, and countless constituents of the traffic / mobility ideal are next discussed. Current trials in growing VANET simulators, and the description of resolutions adopted so distant are subjects of a distinct serving, in that subjects considering the presentation of disparate simulation instruments are presented.

\section{General features of VANET Simulators}

VANET simulators can be categorized as microscopic or macroscopic. Microscopic traffic simulators emphasize innate deeds of individual vehicles by representing the velocity and locale of every single vehicle at a given moment. This kind of simulation is exceptionally helpful for studying localized traffic contact, but it comes alongside the worth of decreased scalability.

\section{Simulator architecture}

A VANET simulator has two main components: a web constituent, capable of simulating the deeds of a wireless web, and a vehicular traffic constituent, able to furnish a precise mobility ideal for the nodes of a VANET

\section{Communication models}

Communication models highlight the data flows amid vehicles and are of distinct attention after vehicles move alongside elevated comparative speeds. VANET requests are altered by wireless networking aspects such as transmission stay, packet defeat or web admission scheme.

\section{Mobility Models}

Mobility models emphasize innate "behavior" of individual vehicles by representing the velocity and locale of every single vehicle at a given moment. This kind of simulation is exceptionally helpful for studying localized traffic interactions. Vehicular traffic mobility models have been learned for a long time.

After selecting a mobility ideal for a VANET simulator, the intricacy and the accuracy are the most vital subjects demand to be considered. The benefit of employing a extremely convoluted and precise mobility ideal, jointly alongside the presentation penalty, ought to be prudently assessed, keeping in mind the intention of the VANET simulator and the kind of requests for that it is designed.

\section{Implementation issues}

The integration amid the mobility ideal and the web contact ideal is safeguarded in the design phase. The integration is implicitly attained as the developer's onset alongside this integration in mind.

Maps

A digital chart is needed in nearly each VANET application. Every single vehicle that is portion of the arrangement ought to have admission to such a digital map. A extremely accepted option for digital charts utilized by VANET simulators is TIGER - Topologically Consolidated Geographic Encoding and Referencing (2008), that is freely-available, actual digital charts for the USA.

\section{Open Street Map}

Open Street Map (OSM) is a cooperative undertaking to craft a free editable chart of the world. Two main steering powers behind the formation and development of OSM have been limits on use or potential of chart data across far of the globe and the advent of inexpensive handy satellite exploration devices. Crafted by Steve Coast in the UK in 2004, Openstreet chart has grown to above 1 million registered users, who can amass data employing GPS mechanisms, aerial photography, and supplementary free origins.

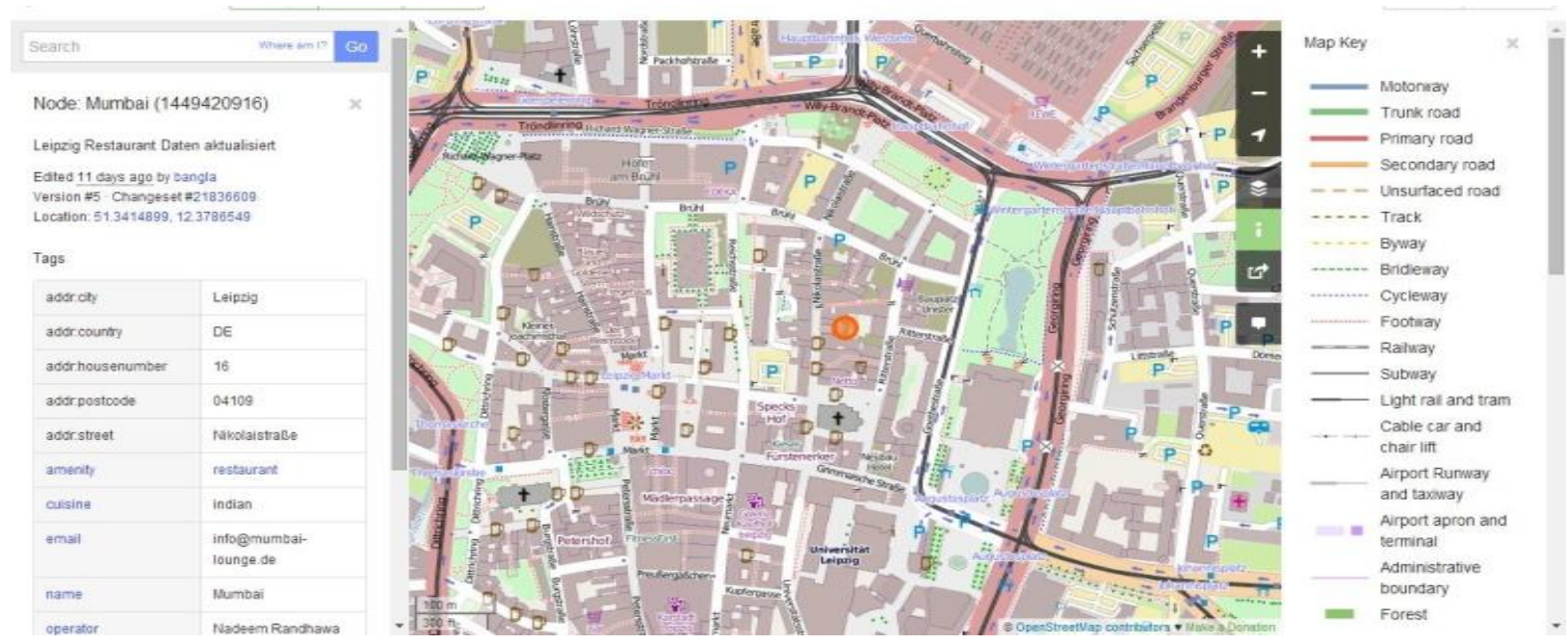

Figure 1: Open Street Map

In the Figure 1 we have shown open street map of a city so that we will be able to calculate various parameters. 


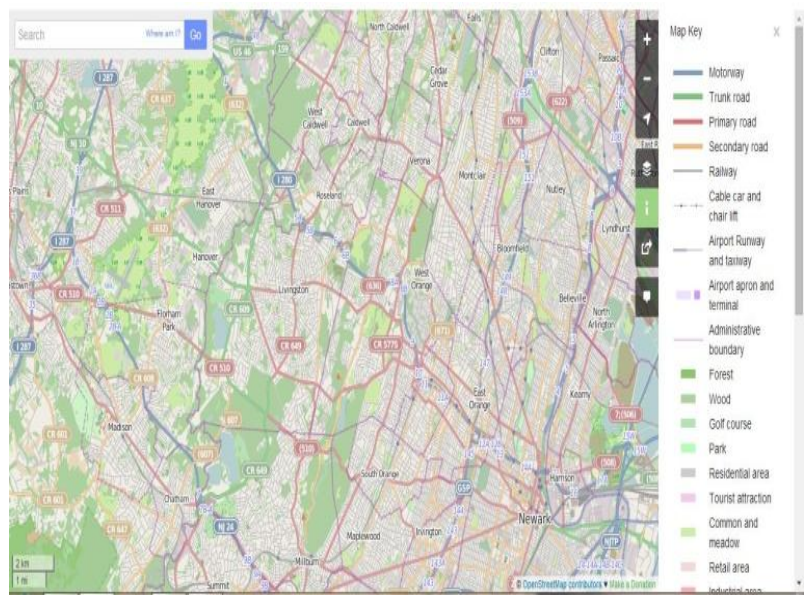

Figure 2: Features of OSM

In Figure 2 we have shown features of Open Street Map which will help in calculating various parameters.

\section{Elements}

Elements (also data primitives) are the basic components of Open Street Map conceptual data model of the physical world. They consist of

1 nodes (defining points in space),

2 ways (defining linear features and area boundaries), and

3 Relations (which are sometimes used to explain how other elements work together).

\section{Node}

A node embodies a specific point on the earth's external described by its latitude and longitude. Every single node embodies at least an id number and a pair of coordinates. Nodes can be utilized to delineate standalone point features.

\section{Way}

A method is an arranged catalog of amid 2 and 2,000 nodes that delineate a polyline. Methods are utilized to embody linear features such as streams and roads. Methods can additionally embody the borders of spans (solid polygons) such as constructions or forests. In this case, the way's early and last node will be the same. This is shouted a "closed way".

\section{Relation}

A relation is a multi-purpose data construction that documents a connection amid two or extra data agents (nodes, methods, and/or supplementary relations). Examples include: A path relation that catalogs the methods that form a main (numbered) freeway, a series path, or a bus route.

\section{Tag}

All kinds of data agent (nodes, methods and relations) can have tags. Tags delineate the meaning of the particular agent to that they are attached. A tag consists of two free format text fields; a 'key' and a 'value'. Every single of these are Unicode strings of up to 255 characters. For example, highway=residential defines the method as a road whose main purpose is to give admission to people's homes.

\section{RESULTS}

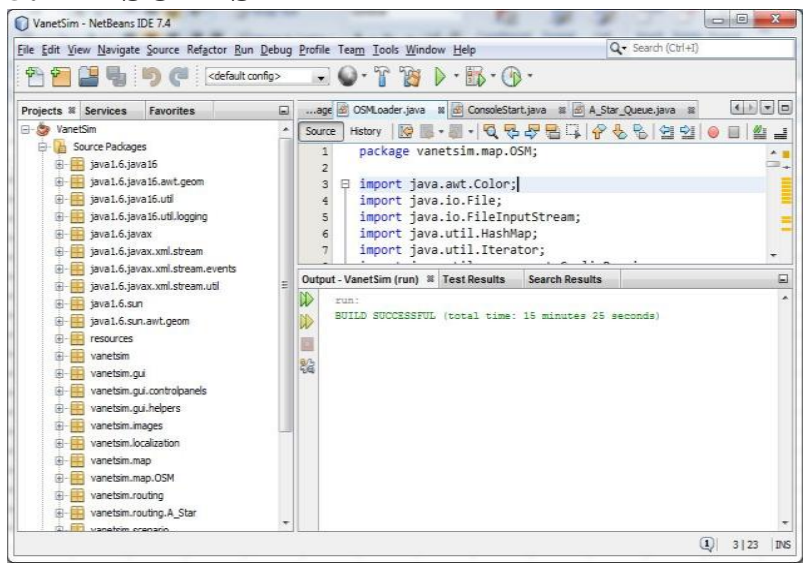

Figure 3: VANET SIM with A* Algorithm

In Figure 3 we have shown simulator VANET SIM which will perform calculation using A* Algorithm

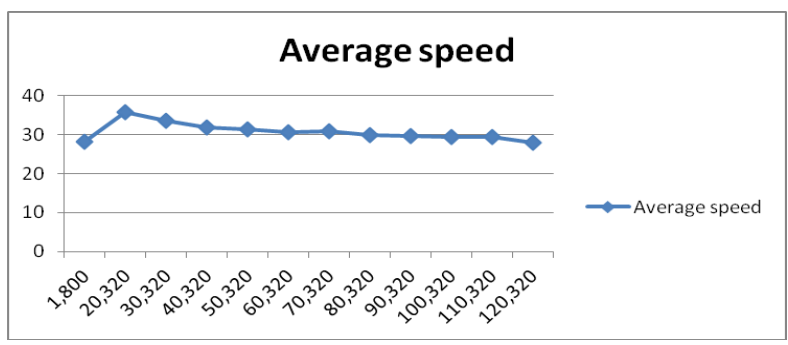

Figure 4: No of Active Vehicles

In Figure 4 we have shown no of active vehicles present and their average speed

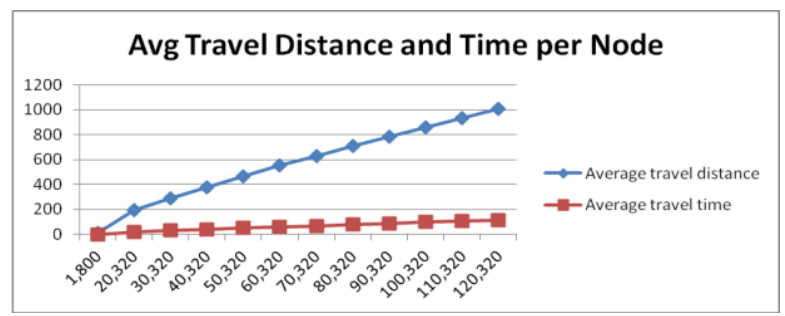

Figure 5: Average Distance and Time

In Figure 5 we have shown average distance travelled by vehicle (node) per unit time.

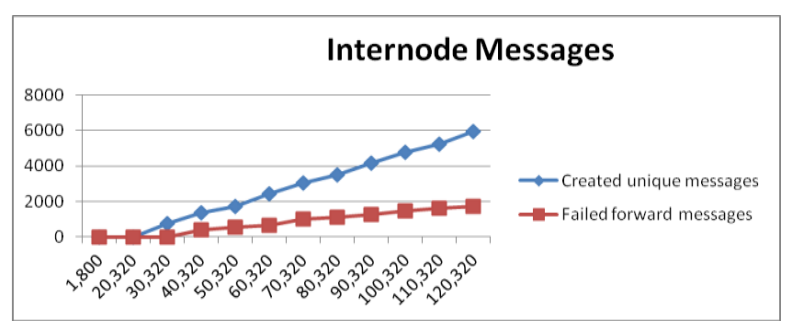

Figure 6: Internode Messages

In Figure 6 we have shown Inter node message passed between nodes

\section{CONCLUSION}

Vehicular ad hoc webs (VANETs) are anticipated to prop a colossal spectrum of mobile distributed requests that scope 
from traffic alert dissemination and vibrant path arranging to context-aware advertisement and file sharing. In VANETS main concern is whether the presentation of VANET routing protocols can gratify the throughput and stay necessities of such applications. Our work focuses on VANET routing in city-based scenarios. We have been successful in achieving the following objectives that were set for this research work

1. Have industrialized a VANET-Simulation scenario for upholding assorted VANET specific applications. The colossal spectrum of mobile distributed requests scope from traffic alert dissemination and vibrant path arranging to context-aware advertisement and file sharing.

2. Made an simulation setup to permit colossal number of mobile nodes (vehicles), Thinking the colossal number of nodes that give in these webs and their elevated mobility.

3. Designed and requested an enhanced ideal for Intersection Instituted VANET Routing on actual Metropolis Roads(maps) from real globe cities. Metropolis Charts are imported from XML files.

4. Generation of Real period Vehicular Traffic that can be utilized to examination the VANET, the arrangement will use the Micro-simulation of every single vehicle in the scenario. Every single vehicle will be simulated individually

5. Enabled random choices by the vehicle in the Microsimulation of every single vehicle hence vehicle can seize decisions on its own making road traffic is as realistic as possible.

6. Finally, they additionally assessed the presentation best in words of average transport rate, no of alert vehicles, Average speed of every single vehicle.

\subsection{Future Scope}

Traffic jams and traffic accidents have come to be a main concern in present society. VANET (vehicle ad hoc network) is a growing appealing request to resolve such problems. VANET Routing is accountable for configuring data transmission trail amid vehicles dispatching and consenting memo by employing car node as relay node. As car node is of mobility, it is tough to uphold contact connectivity amid two acquaintance vehicles.

\section{REFERENCES}

[1] Portmann, Marius, and Asad Amir Pirzada. "Wireless mesh networks for public safety and crisis management applications." Internet Computing, IEEE 12, no. 1 (2008): 18-25.

[2] Hu, Yih-Chun, Adrian Perrig, and David B. Johnson. "Ariadne: A secure on-demand routing protocol for ad hoc networks." Wireless networks 11, no. 1-2 (2005): 2138.

[3] Suwart, Jan. "Wireless Ad Hoc Networks: Limitations, Applications and Challenges." (2008).

[4] Stenbom, Håkan. "Optimization of Remote ServiceSolution for large installations: Wireless LAN and WAN for ABB Robotics." (2011).

[5] Ramachandran, Lakshmi, Sangheethaa Sukumaran, and Surya Rani Sunny. "An Intersection Based Traffic Aware Routing With Low Overhead in VANET."International
Journal of Digital Information and Wireless Communications (IJDIWC) 3, no. 2 (2013): 50-56.

[6] Tavakoli, Rasool, and Majid Nabi. "TIGeR: A TrafficAware Intersection-Based Geographical Routing Protocol for Urban VANETs." Vehicular Technology Conference (VTC Spring), 2013 IEEE 77th. IEEE, 2013.

[7] Sukumaran, Sangheethaa, Lakshmi Ramachandran, and Surya Rani Sunny. "STUDY OF VARIOUS GEOGRAPHIC ROUTING PROTOCOLS IN VANET."International Journal of Research in Wireless Systems 2, no. 1 (2013).

[8] Al Hanbali, Ahmad, Eitan Altman, and Philippe Nain. "A survey of TCP over ad hoc networks." IEEE Communications Surveys and Tutorials 7, no. 1-4 (2005): 22-36.

[9] Bruno, Raffaele, Marco Conti, and Enrico Gregori. "Mesh networks: commodity multihop ad hoc networks." Communications Magazine, IEEE 43, no. 3 (2005): 123-131.

[10] Li, Fan, and Yu Wang. "Routing in vehicular ad hoc networks: A survey."Vehicular Technology Magazine, IEEE 2, no. 2 (2007): 12-22.

[11] Samara, Ghassan, Wafaa AH Al-Salihy, and R. Sures. "Security Analysis of Vehicular Ad Hoc Nerworks (VANET)." In Network Applications Protocols and Services (NETAPPS), 2010 Second International Conference on, pp. 55-60. IEEE, 2010.

[12] Boukerche, Azzedine, Horacio ABF Oliveira, Eduardo F. Nakamura, and Antonio AF Loureiro. "Vehicular ad hoc networks: A new challenge for localization-based systems." Computer communications 31, no. 12 (2008): 2838-2849.

[13] Mishra, Bharati, Priyadarshini Nayak, Subhashree Behera, and Debasish Jena. "Security in vehicular adhoc networks: a survey." In Proceedings of the 2011 International Conference on Communication, Computing \& Security, pp. 590-595. ACM, 2011.

[14] Raya, Maxim, and Jean-Pierre Hubaux. "The security of vehicular ad hoc networks." Proceedings of the 3rd ACM workshop on Security of ad hoc and sensor networks. ACM, 2005.

[15] MEGHDADI, Vahid. "Vehicular Ad-Hoc Networks (VANET) applied to Intelligent Transportation Systems (ITS)."

[16] Meshkova, Elena, Janne Riihijärvi, Marina Petrova, and Petri Mähönen. "A survey on resource discovery mechanisms, peer-to-peer and service discovery frameworks." Computer networks 52, no. 11 (2008): 2097-2128.

[17] Shi, Jingmin, et al. "There is a Will, There is a Way: A New Mechanism for Traffic Control Based on VTL and VANET." High Assurance Systems Engineering (HASE), 2015 IEEE 16th International Symposium on. IEEE, 2015.

[18] Sahu, Pratap Kumar, Eric Hsiao-Kuang Wu, Jagruti Sahoo, and Mario Gerla. "BAHG: back-bone-assisted hop greedy routing for VANET's city environments." Intelligent Transportation Systems, IEEE Transactions on 14, no. 1 (2013): 199-213. 